(C) 1981. The Genetical Society of Great Britain

\title{
THE SELECTIVELY OPTIMAL PHENOTYPES OF THE COXAL CHAETAE IN DROSOPHILA MELANOGASTER
}

\author{
P. D. S. CALIGARI* \\ Department of Genetics, University of Birmingham, Birmingham B15 2TT \\ Received 18.xi.80
}

\section{SUMMARY}

\begin{abstract}
Variation in coxal chaeta number on each of the three pairs of legs of Drosophila melanogaster is investigated, alongside that for sternopleural chaeta number, and the selectively optimal phenotypes are estimated employing the method developed by Caligari and Mather, 1980. The results point to coxal chaeta number being subject to stabilizing selection as was inferred previously for sternopleural chaeta number.
\end{abstract}

\section{INTRODUCTION}

IN an earlier paper, Caligari and Mather, 1980, reported an investigation into the genetical architecture of two characters in Drosophila melanogaster. The two characters were defined as $M$, the mean sternopleural chaeta number averaged over flies raised at two temperatures $\left(18\right.$ and $\left.25^{\circ} \mathrm{C}\right)$ and $S$, half the difference between the average chaeta numbers at the two temperatures. It was demonstrated that the results for both characters were consistent with them being subject to a past history of stabilizing selection. Furthermore, a method was developed and applied to the results which enabled the phenotypes of maximum dominance to be estimated and these, it was argued, were the optimal phenotypes towards which the selection had been acting. The aim of the present paper is to utilize this general method to investigate the selective optima displayed by the chaetae carried on the coxae of the three pairs of legs of Drosophila melanogaster.

\section{THE EXPERIMENT}

The 16 inbred lines used in the present experiment were derived by sib-mating from the "Texas" cage population and are 16 of the 18 lines described by Caligari and Mather (loc. cit). These 16 lines were crossed to make a half-diallel set of matings, thus giving 136 cultures, which were raised on standard medium in $2.5 \mathrm{~cm} \times 7.5 \mathrm{~cm}$ glass vials at $25^{\circ} \mathrm{C}$. The whole set of matings was replicated to give 272 cultures in total. From the progeny in each of these cultures 5 females were taken at random and scored for the number of coxal chaeta on each of the three pairs of legs, front $(\mathrm{F})$, middle $(\mathrm{M})$ and rear $(\mathrm{R})$, at the same time the numbers of sternopleural chaetae (St) were also recorded. The basic datum for each character was the chaeta number summed over the two sides of the fly and averaged over the 5 flies scored.

* Present Address: Scottish Crop Research Institute, Pentlandfield, Roslin, Midlothian EH25 9RF. 
Since interest centres on the properties of the population, the question arises as to whether the inbred lines under study represent a fair sample of the genes present in the population from which they were derived. This can be investigated by comparing the results obtained from the progenies of single pair matings taken directly from the population with those from the inbreds themselves as well as those from progeny produced in a diallel mating scheme between the inbred lines. As far as the means are concerned, if the lines are a fair sample of the population, the estimates from all three sources should agree with each other within the limits of sampling. With regard to the variances, however, the three are not expected to be the same since the variance between the inbred lines will reflect the full additive genetic effects of the loci but will be unaffected by the dominance relations, while the other two will not only express no more than a quarter of the additive genetic effects but will also be affected by the dominance properties of the genes. However, if the inbred lines are a fair sample of the population, the diallel results will approximate to a reconstruction of that population, approximate in that although the diallel will give Hardy-Weinberg frequencies for each individual locus a sample of, at most, 16 lines can no more than approach the frequencies and combinations of genes possible in the population. Thus the agreement between the variances of the population itself and the reconstituted one cannot be expected to be exact, but the two should bear a close resemblance to one another and differ from the variance of inbred lines in the same way.

The three estimates of the means of the two characters $M$ and $S$ reported earlier by Caligari and Mather are given in table 1 and can be seen to be

TABLE 1

Sternopleural chaetae

$\overbrace{M}^{S} \mathrm{St}$

Pop. mean

Pop. variance

Inbred Line mean

Inbred Line

Variance (I)

Reconstituted

Pop. mean

Reconstituted

Pop. variance (R.P.)

I/RP $\begin{array}{lll}18.81 & 0.53 \quad 18.28\end{array}$

$\begin{array}{lll}0.614 & -0.099 & 0.676\end{array}$

$19.15 \quad 0.45 \quad 18.48$

$\begin{array}{lll}5.422 & 0.501 & 7.577\end{array}$

$\begin{array}{lll}19.15 & 0.27 \quad 18.22\end{array}$

0.928

0.057

$8 \cdot 8$

$1 \cdot 108$

$6 \cdot 8$

$\begin{array}{ccc}\overbrace{F} & M & R \\ - & - & - \\ - & - & - \\ 24.56 & 21.19 & 15.18 \\ 6.413 & 2.339 & 0.973 \\ 24.91 & 21.35 & 15.44 \\ 1.343 & 0.494 & 0.131 \\ 4.8 & 4.7 & 7.4\end{array}$

in close agreement with each other. The variances of the population and the reconstituted population are also given and are clearly of the same order of magnitude as one another, while those of the inbreds themselves are much larger: indeed, both are greater than the anticipated factor of 4 (as shown by the ratio of I/R.P.) indicating the presence of dominance with the dominant allele the more common, a point which will be returned to later.

This pattern is repeated for St of the present experiment. Results for the coxal chaetae from single pair matings raised under similar conditions to those of the experiment are not available. However, it is possible to 
compare the results from the inbred lines with those from the reconstituted population. For each set of coxal chaetae the two estimates of the mean show little evidence of significant differences while the ratios of I to R.P. closely parallel those for the sternopleurals with all of them exceeding the factor of 4-a parallel which cannot be simply attributed to chance. In other words not only does the evidence from the sternopleurals provide every indication of the inbred lines constituting a fair sample of the genes present in the original population, as was concluded by Caligari and Mather, but the results for the coxal chaetae also lead to the same conclusion: indeed, they reinforce it.

\section{RESULTS AND ANALYSIS}

Having established that the inbred lines provide a fair sample of the genes in the population it is worth examining more closely the relationships between the variances, the genetical components of which are presented in table 2. The notation used is that of Mather and Jinks (1977) where

TABLE 2

Results from the analyses of sternopleural chaetae $(S t)$ and from those of front $(F)$, middle $(M)$ and rear $(R)$ coxal chaetae

St

Genetical components of variances

$\begin{array}{cc}D_{P} & 7.577 \\ D_{W} & 5 \cdot 440 \\ D_{R} & 4 \cdot 262 \\ H_{R} & 0.678 \\ \sqrt{D_{P} \cdot D_{R}} & 5 \cdot 683 \\ \sqrt{H_{R} / D_{P}} & 0.295 \\ u \text { and } v & 0.88,0 \cdot 12\end{array}$

$W_{r}+V_{r} /$ recurrent parent

$$
-b_{1} / 2 b_{2}
$$

$16 \cdot 039$

$\mathrm{F}_{1}-\mathrm{M} . \mathrm{P} . /$ non-recurrent parent

\begin{tabular}{l}
\multicolumn{1}{c}{$\hat{b}$} \\
M.S. for scatter \\
Rep. error var. \\
Phenotype of max. \\
$\quad$ dominance. \\
Mean of parents \\
Difference \\
$* \mathbf{P}=5-1$ per cent. \\
$* * \mathbf{P}=1-0 \cdot 1$ per cent.
\end{tabular}

$-0 \cdot 172$

$0 \cdot 466$

$0 \cdot 240$

$\overbrace{\mathrm{F} \quad \mathrm{M}}^{\text {Coxals }}$

$\begin{array}{ccc}6.413 & 2.339 & 0.973 \\ 5 \cdot 750 & 2 \cdot 071 & 0.662 \\ 5 \cdot 191 & 1.932 & 0.500 \\ 0.718 & 0 \cdot 181 & 0.103 \\ 5 \cdot 770 & 2 \cdot 126 & 0.697 \\ 0.335 & 0.278 & 0 \cdot 325 \\ 0.68,0 \cdot 32 & 0 \cdot 82,0 \cdot 18 & 0 \cdot 88,0 \cdot 12\end{array}$

$22 \cdot 471$

$21 \cdot 318$

$16 \cdot 056$
$-0 \cdot 085$
0.641
$-0 \cdot 096$
$0 \cdot 285$
$0 \cdot 179$

$-0 \cdot 217$

$0 \cdot 158$

$0 \cdot 118$

$\begin{array}{clrr}17.70 \pm 0.28 & 26 \cdot 77 \pm 0.47 & 22 \cdot 05 \pm 0 \cdot 34 & 15 \cdot 80 \pm 0 \cdot 10 \\ 18.48 \pm 0.70 & 24 \cdot 56 \pm 0.65 & 21 \cdot 19 \pm 0.40 & 15 \cdot 18 \pm 0 \cdot 26 \\ -0.78 \pm 0.75 & 2 \cdot 21^{* *} \pm 0.80 & 0 \cdot 86 \pm 0.52 & 0.62^{*} \pm 0.28\end{array}$

$D p=\Sigma\left(4 u v d^{2}\right), \quad D_{W}=\Sigma\{4 u v d[d+(v-u) h]\}, \quad D_{R}=\Sigma\{4 u v[d+(v-u) h]\}^{2}$ and $H_{R}=\Sigma\left(16 u^{2} v^{2} h^{2}\right)-\Sigma$ indicating summation over all relevant loci, $u$ and $v$ representing the frequencies of the increasing and decreasing alleles respectively while $d$ is taken as the genetically additive and $h$ the dominance effects of a locus. The results for the sternopleural and three sets of coxal 
chaetae are remarkably similar in the features they exhibit. In all cases, $D_{P}>D_{W}>D_{R}$ with $D_{W}$ close to the geometric mean of $D_{P}$ and $D_{R}$. In other words they all indicate that dominance must be present at individual loci with the dominant alleles generally more common than their recessive counterparts, thus making $(v-u) h$ negative and giving the observed relationship between $D_{P}, D_{W}$ and $D_{R}$. Also, since $D_{W}$ is near to $\sqrt{D_{P} . D_{R}}$ for each character it suggests that there is little variation between the relevant loci in their ratios of $(v-u) h$ to $d$. Thus for all the characters it appears that the dominance is of an ambi-directional nature. This conclusion is further supported, although the graphs are not presented here, when $W_{r}+V_{r}$ (variation in which reflects non-additive genetic effects) is plotted against the expression of the character in the recurrent parent of the array. For each of the characters the relationship is parabolic and reasonably symmetrical indicating that at some loci it is the allele which has an increasing effect on the character that is dominant while at others the decreasing allele is dominant, the two sorts of loci being approximately equally frequent. The minimum value of $W_{r}+V_{r}$ represents the array displaying maximum dominance and, if a second degree multiple regression of the type $y=a+b_{1}(x-\bar{x})+b_{2}\left(x^{2}-\bar{x}^{2}\right)$ is fitted, the value of $x$ corresponding to the point of minimum $W_{r}+V_{r}$ can be estimated as $-b_{1} / 2 b_{2}$. Such estimates are presented in table 2 where it can be seen that for each character the estimated phenotype of maximum dominance is close to the mean of the parent lines used.

The four sets of chaetae show very similar patterns of results not only to one another but also to the two characters reported by Caligari and Mather. Indeed, the agreement between the results for the sternopleurals and its counterpart, character $M$, is remarkably good, particularly when allowance is made for the different numbers of inbred lines used in the two studies and the fact that only one temperature was used in the present experiment whereas character $M$ is averaged over two. Thus it would appear that the three sets of coxal chaetae are displaying properties very similar to those of the characters reported earlier. In other words, the more common alleles seem again to be the dominant ones and that it is approximately equally common for them to be the increasing or the decreasing alleles.

Given the similarity in the underlying gene systems, the predictions concerning the regression of $F_{1}$ minus Mid-Parent (M.P.) on the mean of the non-recurrent parent should hold good for the present characters and hence allow the phenotypes of maximum dominance to be estimated. When such regressions were estimated, the results given in the lower portion of table 2 were obtained. Within each character the slopes of the regression for the different arrays proved not to show any significant heterogeneity and were therefore pooled to give a single joint estimate of $b$. In accordance with expectations the joint regressions were negative and the mean squares (M.S.) for scatter about the regression lines were greater than the appropriate replicate error variances. Using these regressions the phenotype of maximum dominance was estimated for each character. As with the estimates from second degree statistics, the values obtained were close to the mean of the parents although in two cases, $F$ and $R$, the differences are, in fact, formally significant. It should be noted that, in general, the present estimates are closer to their appropriate means than those from the $W_{r}+V_{r}$ 
regressions, which must simply reflect the increased precision obtained by using first degree statistics.

Thus here, as for the earlier characters, the dominant alleles tend to be common ones and the phenotype of maximum dominance is close to the mean of the inbred lines and hence, of course, to that of the population for all the characters studied. It should be noted that the regression method used to estimate the phenotype of maximum dominance depends only on the empirical finding that the dominant alleles tend to be more common than their recessive counterparts. This may have arisen in either of two ways. Either the alleles became common because they are dominant or they became dominant because they are common. Which ever is the case it can hardly be other than the result of selection and the phenotype of maximum dominance can then be regarded, as argued by Caligari and Mather, as representing the selectively optimal phenotype. Indeed, this argument gains even greater support if two further estimates are considered. The first is the average degree of dominance, measured as $\sqrt{{ }^{H} R / D_{P}}$, (table 2); it can be seen that for all four characters this is remarkably constant. The second estimation is of the average gene frequencies which are given as $u$ and $v$ in the table although, of course, it is not possible to specify which is which since they are obtained from an estimate of $4 u v$ given by $H_{R} / H_{1}$, where $H_{1}=D_{R}-2 D_{W}+D_{P}+H_{R}$. It is difficult to obtain any estimate of the error variance attached to these frequencies; they all, however, appear to deviate from $u=v=0 \cdot 5$, with St, $\mathrm{M}$ and $\mathrm{R}$ showing extremely close similarity. Such constancy of the dominance ratios and gene frequencies over the four characters leave little doubt that they reflect the past actions of selection on the population.

\section{INTERDEPENDENCE OF THE CHARACTERS}

Before, however, the results are accepted on their face value as showing four independent characters all agreeing well with earlier predictions, two cautionary notes need to be added. The first is statistical and is in two parts, which are: (a) the results presented are from a half-diallel mating scheme in which it is known that internal correlations can arise which could affect the tests of significance within a character, and (b) the four sets of chaetae were all scored on the same flies and therefore environmental as well as developmental effects are likely to be common which will tend to add an environmental component to the correlations between them.

The second point of caution arises from the underlying genetical basis of coxal chaeta number. Mather and Hanks (1978) put forward the hypothesis that the coxal chaetae are controlled by three classes of genes: $\alpha$ genes which are active on all three pairs of legs, $\beta$ genes which are active on the middle and front legs, but not on the rear, and $\gamma$ genes which are active on the front legs only, the activity of the different classes of genes being controlled by an underlying regulatory gradient. This hypothesis gained strong suport when the detailed predictions to which it gave rise were tested against the observed results of selection experiments (Hanks and Mather, 1978). On this basis the chaeta numbers of the rear legs (R) reflect the action of $\alpha$ genes, the middle legs $(\mathrm{M})$ reflect that of $\alpha+\beta$ genes while the front (F) display the combined effects of $\alpha+\beta+\gamma$. Clearly the three sets of chaetae all have the $\alpha$ genes in common, while $\mathrm{M}$ and $\mathrm{F}$ share 
not only $\alpha$ genes but also $\beta$ genes and this will, of course, lead to correlations between the expressions of the character on the different legs.

Assuming, with Mather and Hanks (l.c.), that the expressions of the different genes do not change with legs, the genetical interdependence can be removed by redefining the characters to show the effects of the three classes, i.e., $\alpha=\mathrm{R}, \beta=\mathrm{M}-\mathrm{R}$ and $\gamma=\mathrm{F}-\mathrm{M}$. It should be noted, however, that the comparisons which separate the classes of genes are not orthogonal to one another and so will, in fact, themselves generate statistical independence, as can be seen in table 3 , and hence require cautious interpretation.

Bearing in mind these notes of caution it would nevertheless seem worthwhile removing the genetical interdependence between the three sets of coxae to see whether this materially affects the interpretation. When the effects of the three classes of gene are separated and the analyses repeated, the results in table 3 are obtained. It is immediately apparent from this table that all the features detailed for the original characters are present for the three classes of gene and would all, therefore, lead to the conclusion of ambi-directional dominance and selective optima close to the mean of the parent lines.

\section{Conclusions}

The results from the analysis of sternopleural chaeta number in the present experiment closely parallel those found by Caligari and Mather (1.c.). Thus, as previously, it would appear that the results are entirely consistent with a character subject to stabilizing selection with a mean very

TABLE 3

Results from the analyses of the three classes of genes, $\alpha, \beta$ and $\gamma$ controlling coxal chaetae number
$\alpha$
$(\mathbf{B})$
$\stackrel{\beta}{\beta}(\mathrm{M}-\mathrm{R})$
$\stackrel{\gamma}{(\mathrm{F}-\mathrm{M})}$

Genetical components of variances

$\begin{array}{cccc}D_{P} & 0.973 & 1.546 & 1.721 \\ D_{W} & 0 \cdot 662 & 1.275 & 1.557 \\ D_{R} & 0.500 & 1.102 & 1.405 \\ H_{R} & 0 \cdot 103 & 0.032 & 0.230 \\ \sqrt{D_{P} \cdot D_{R}} & 0.697 & 1.305 & 1.555 \\ \sqrt{H_{R} / D_{P}} & 0 \cdot 325 & 0.144 & 0 \cdot 365 \\ u \text { and } v & 0 \cdot 88,0 \cdot 12 & 0.93,0.07 & 0.94,0.06\end{array}$

$W_{r}+V_{r} /$ recurrent parent $-b_{1} / 2 b_{2} \quad 16 \cdot 056$

$11 \cdot 710$

$2 \cdot 513$

$\mathrm{F}_{1}-\mathrm{M} . \mathrm{P} . /$ non-recurrent parent

\begin{tabular}{lrrr}
\multicolumn{1}{c}{$\hat{b}$} & $-0 \cdot 217$ & $-0 \cdot 159$ & $-0 \cdot 143$ \\
M.S. for scatter & $0 \cdot 158$ & $0 \cdot 354$ & 0.555 \\
Rep. error var. & $0 \cdot 118$ & $0 \cdot 294$ & $0 \cdot 427$ \\
Phenotype of max. & & & \\
$\quad$ dominance & $15 \cdot 80 \pm 0 \cdot 10$ & $5 \cdot 68 \pm 0 \cdot 20$ & $4 \cdot 09 \pm 0 \cdot 26$ \\
Mean of parents & $15 \cdot 18 \pm 0 \cdot 26$ & $5 \cdot 01 \pm 0 \cdot 34$ & $3 \cdot 36 \pm 0 \cdot 37$ \\
Difference & $0 \cdot 62^{*} \pm 0 \cdot 28$ & $-0 \cdot 33 \pm 0 \cdot 39$ & $0 \cdot 73 \pm 0 \cdot 45$ \\
$* \mathrm{P}=5-1$ per cent. & & &
\end{tabular}


close to the optimum phenotype. The similarity of the behaviour of the coxal chaetae to that of the sternopleurals is striking, whether the analysis is carried out on the three legs separately or in terms of the $\alpha, \beta$ and $\gamma$ genes. This close similarity makes unreasonable any attempt to attribute it to chance. It could be due to one or other of two possible causes. The first possibility is that the genes controlling all the characters are to a large extent pleiotropic in their action and therefore the results are simply reflecting the properties of a single gene system. However, although for the three sets of coxal chaetae there are genes which are pleiotropic on all legs $(\alpha)$, others which are pleiotropic on just two $(\beta)$ and still others which are not pleiotropic at all $(\gamma)$, when these classes of genes are examined separately the similarities remain despite their independent behaviour under artificial selection (Hanks and Mather, 1.c.). Also, among the inbred lines, there is no evidence of a significant correlation between, for example, the sternopleurals and front coxals. Thus there is little support for the existence of the wide-ranging pleiotropy which would be necessary to account for the present results. This, therefore, leaves the second possibility which is that the similarities are due to the different characters all having been subject to a similar type of selection pressure. All the indications from the genetical architecture and estimated optima are that the type of selection they have been under in the past was stabilizing, resulting in the mean and optima being close together.

Acknowledgements.-I gratefully acknowledge the continuing help and advice of Professor Sir Kenneth Mather. The financial support of the Agricultural Research Council made this work possible.

\section{REFERENCES}

CALIGARI, P. D. S., AND MATHER, K. 1980. Dominance, allele frequency and selection in a population of Drosophila melanogaster. Proc. Roy. Soc. Lond. B., 208, 163-187.

HANKS, M. J., AND MATHER, K. 1978. Genetics of coxal chaetae in Drosophila melanogaster. II. Responses to selection. Proc. Roy. Soc. Lond. B., 202, 211-230.

MATHER, K., AND JINKS, J. L. 1977. Introduction to biometrical genetics. Chapman and Hall, London.

MATHER, K., AND HANKS, M. J. 1978. Genetics of coxal chaetae in Drosophila melanogaster. I. Variation in gene action. Heredity, 40, 71-96. 